\title{
Chong-Chet Anterior Scoring Technique for the Correction of Prominent Ears: Results in 30 Patients
}

\author{
Nazan Sivrioglu, Saime Irkoren", Banu Aksoy, Eray Copcu \\ Department of Plastic and Reconstructive Surgery, Faculty of Medicine, Adnan Menderes University, Aydin, Turkey. \\ Email: *saimeirkoren@hotmail.com
}

Received December 21 ${ }^{\text {st }}, 2011$; revised January $25^{\text {th }}, 2012$; accepted February $26^{\text {th }}, 2012$

\begin{abstract}
A lot ofotoplasty techniques have been developed during the past years. However, there is no single perfect technique which is worldwide accepted. We present our assessment of a Chong-Chet anterior scoring technique for the correction of prominent ears which we applied in 30 patients between 2005 and 2010. This study is a report of creating a natural fold of cartilage using Chong-Chet anterior scoring technique by cartilage incision at the border between the antihelix and scapha, with medial scoring of the scapha and lateral scoring of the antihelix. Sutures were used to maintain the created fold. There were no major complications at the 1-year follow-up evaluation. None of the patients developed a recurrence, or retro protrusion of the helix which required a second operation for correction. The procedure is a simple surgical technique. The reconstructed ear shows reliable results in stability, size and normal convolutions. Furthermore, sharp cartilaginous ridges and hidden helix are avoided.
\end{abstract}

Keywords: Chong-Chet; Anterior Scoring; Prominent Ear

\section{Introduction}

A prominent ear, so called a protruding or "lop" ear, is the most common congenital deformity of the external ear. It is relatively common among the Caucasian population, with an incidence of about 5\%. Prominent ear is characterized by an increase in the cephaloauricular angle, which occurs because of an immature antihelical fold; an excessive conchal cartilage; or abnormal attachment of the auricle to the side of the head, alone or in combination.

Numerous otoplasty techniques have been developed to optimize the appearance and position of the ear showing that no single ideal technique has been worldwide accepted. 4e22 among otoplasty techniques we can divide them into two groups: the cartilage-incision and the suture-placement procedures. Combined techniques and a large number of surgical techniques or modifications have been explained to improve the cosmetic outcome [1-6]. Scoring of the anterior surface of the auricular cartilage is one of the most commonly used procedures in otoplasty, and we believe it is a very safe technique.

The aim of this article is to present our experience with Chong-Chet anterior scoring procedure.

\section{Materials and Methods}

Between 2005 and 2011 we treated 30 patients (13 males

"Corresponding author. and 17 females) with prominent ears arising from a lack of development or underdevelopment of the antihelical fold and an oversized concha. Considering every ear on the same patient as a variable, a total of 56 procedures have been performed (26 bilateral and 4 unilateral).The patients were between 6 and 38 years old (mean 22). We did not include patients with incomplete clinical data.

We analyzed the age, sex, surgical technique, side effect, the need for a second operation, type of anesthetics, additional procedures, follow-up, complications, and results.

\section{Operative Procedure}

Local anesthesia induced by $0.5 \%$ bupivacaine with 1:200,000 epinephrineswas used. No sedation was applied for adult patients, whereas pediatric patients underwent general anesthesia. We currently perform a modified Chong-Chet procedure. With pressure applied medially to the prominent ear helix, the future antihelical fold is shown on the anterior surface. To correct the antihelix we used a 27-gauge needle, with the tip stained with methylene blue, which is first inserted through the anterior surface on each side of the supposed fold in order to mark it posteriorly. A 3-cm hourglass-shaped strip of posterior auricular skin is excised. The hourglass is drawn between the free margin of the pinna and the cephaloauricular angle. Posterior soft tissue undermining is per- 
formed to expose the cartilage previously dotted line. Cartilage then is incised from the superior antihelical crus to the tail of the future antihelix. After that skin from the anterior aspect of the ear is carefully detached from the cartilage, and the incision is prolonged in a conchal direction to elevate a cartilage flap. The anterior surface of this flap is scored with parallel and crosshatched cuts. This maneuver weakens the ear cartilage, which curves spontaneously.

We stabilize the newly reconstructed antihelix using two to three 4/0 white Vycryl rolling stitches to hold the neo-antihelical fold. Stitch knots have been placed into the rolled cartilage flap (Figures 1(c) and 1(d)). Usually two stitches are enough for a small ear (e.g., a female ear) and three are required for a bigger ear or for dense cartilages. The stitches are positioned in the upper and lower parts of the neo-anti-helix (if necessary the third stitch is placed in the middle).

After the stitches, soft tissue undermining is performed on the upper anterolateral antihelix prominent area along a line continuing the neo-antihelix on the upper helix cartilage to weaken part of the helix curve. Like in the antihelicalarea anterior scoring in the same way weakens this area. When the new antihelix has been created, the skin is closed using four non-absorbable $4 / 0$ mattress stiches placed horizontally.

The ears are dressed by stuffing of the triangular fossa, concha (cavum and cymbum), and scapha with no adherent gauze. This gauze is placed on the posterior incision too. A cotton wool piece covers both the ears, and dry gauze covers the cotton. An elastic bandage holds the dressing in place for 7 days (checked 3 days after surgery).

\section{Results}

There was no hematoma, seroma or infection in any of the patients. None of the patients developed skin necrosis or any visible cartilaginous irregularities. There was mild asymmetry in one patient, with bilateral prominent ears due to under correction of the concha. However, the patient did not undergo reoperation. None of the patients developed keloids. Suture extrusion did not occur in any of the patients during the follow-up period.

No patient developed a recurrence or required a corrective secondary operation. Pre (Figures 1(a) and 1(b)) and postoperative photography (Figures 2(a) and 2(b)) of one illustrative case are presented.

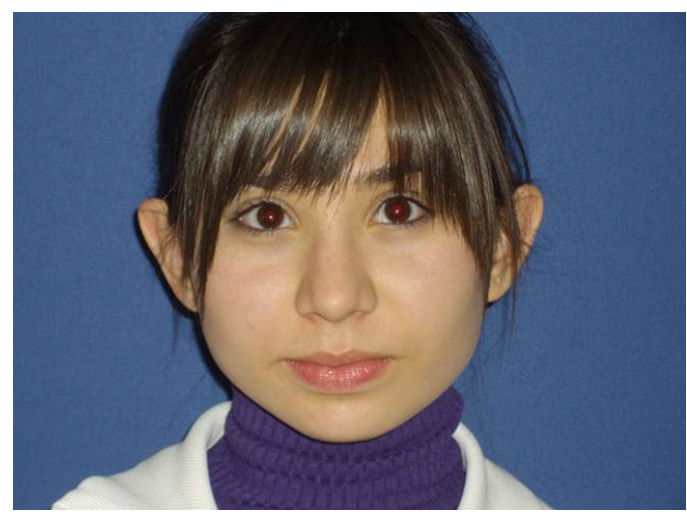

(a)

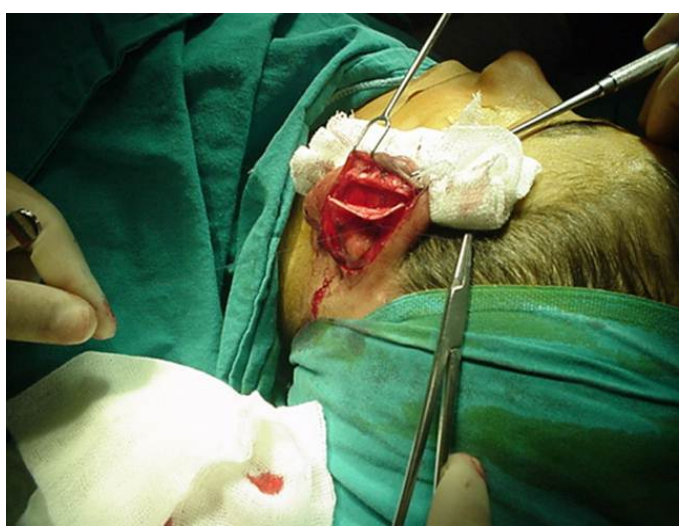

(c)

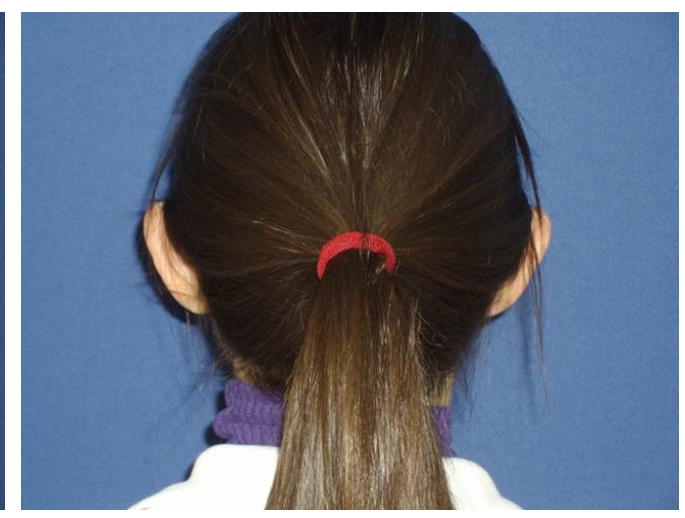

(b)

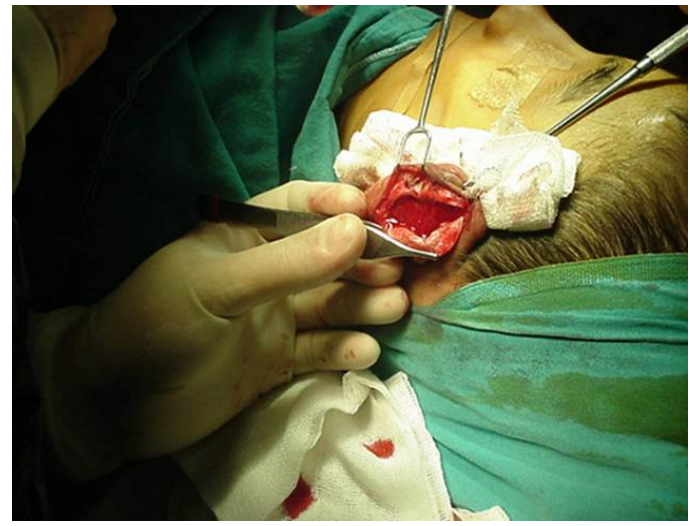

(d)

Figure 1. (a) Front view (preoperative); (b) Back view (preoperative); (c) Intraoperative view applying the suture to the left cartilage; (d) Intraoperative view of the left cartilage after the suture. 


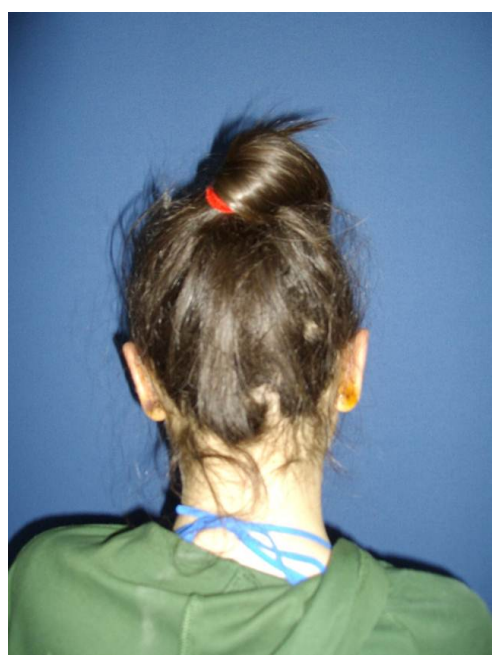

(a)

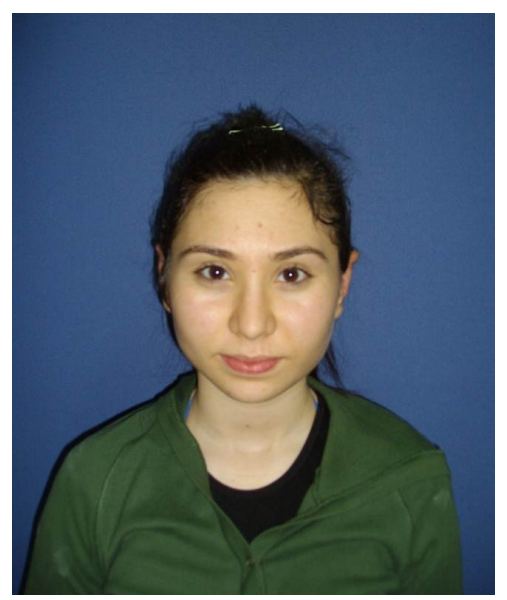

(b)

Figure 2. (a) Back view of the same patient postoperative; (b) Front view of the same patient postoperative.

Patients were followed up at 3, 6, and 12 months followed by yearly intervals for 3 years.

\section{Discussion}

The external ear is a complex structure with great variation between individuals and even between the two sides of the same individual. Proper evaluation is therefore essential to the application of the corrective technique [1].

Over 200 different procedures have been described and proven successful to give high patient satisfaction, making it difficult to address any new technique as necessary [7-9].

These techniques focus on creating a new antihelical fold, reducing the scaphomastoid angle and trimming the concha. Surgical treatments of prominent ear are divided into the anterior and posterior approach $[3,10]$. In the posterior approach, the risk of suture protrusion varied from $0 \%$ to $10 \%$ according to different authors $[5,11]$. None of the patients in this study developed suture protrusion or keloids. The techniques of handling the deformed cartilage can be subdivided into suture-only techniques, cartilage-weakening techniques or a combination of the two [5,11-14]. Furnas described a suture-only technique using non-absorbable sutures based on a post-auricular approach. The problems associated with this technique are secondary relapse because of cartilaginous memory and irregularities created in molding of the conchal floor $[6,8,15]$.

In the 1960s, Mustardè announced a procedure by remodeling of the auricular cartilage and using mattress sutures. However, this procedure had a $7 \%$ relapse rate if the cartilage was too weak, especially if the tissue was thick. This method also includes no change in the conchal cartilage, so the temporoconchal angle remained unchanged. The technique offered by Gersuny 6 in 1903 and revised by Furnas 7 in 1968 consisted of fasten the cartilage to the mastoid periosteum. This allowed the correction of the hypertrophy of the concha without full-thickness resections or incisions into the cartilage, which often had the disadvantage of aesthetically poor stages [15,16-19,20].

Converse described a method of correcting and forming an antihelical fold using a cartilage cutting and suture method. Several full-thickness cuts through the cartilage are carried out in the scaphoid fossa. The problems associated with this method the sharp cartilaginous ridges that can be seen through the thin anterior skin [12,21-24].

Mustardè proposed the use of three to four white silk mattress sutures which are positioned described to make a tube of the auricular cartilage and form the antihelix [15].

Mustardè, even with non-absorbable sutures, it does not always produce stable results. A relapse rate of $7 \%$ for the technique has been reported, which is reasoned to the continual traction exerted by the "cartilage recoil" on the sutures that may cut across the cartilage in some cases. When the methods of Stenström and Mustardèare combined, slowly absorbed sutures can be used because the function of the sutures ends in a few weeks with the development of fibrosis. In cases that rely exclusively on mattress sutures relapse is possible if the knot becomes exteriorized or infected, it requires removal [10,15,19,20, 22]. Based on a study by Gibson and Davis, Stenström suggested a method of weakening the anterior surface of the auricular cartilage by scratching the perichondrium to exploit the elasticity of the cartilaginous tissue on the intact side; this tends to bend the ear in a convex way towards the abraded side [14,20,22]. Stenström's original method involved no alteration to the conchal cartilage, and the temporoconchal angle was unchanged. To create the antihelix, Stenström count exclusively on weakening the cartilage and positioning the transcutaneous sutures 
that held gauze on either side of the newly shaped antihelix for 7 - 8 days. In the 1963 Chong-chet defined an anterior scoring technique with sutures for stabilizing the newly formed antihelix based on a post-auricular approach [14]. We think that it is essential to combine scarification with sutures, as described by Chong-chet particularly in patients with thick cartilage.

In our experience and the surgical technique described, this is a safe way to correct a prominent ear. No major complications were observed, and we achieved good long-term aesthetic results. We performed a retrospective study of patients who underwent otoplasty using a Chong Chet anterior scoring technique combined with post auricular fixation sutures.

In conclusion, the proposed Chong Chet anterior scoring technique is a simple and safe technique that does not cause anterior scarring or skin necrosis. The reconstructed auricle shows reliable results in stability, size and normal convolutions with no recurrence in this study group. In addition, sharp cartilaginous edges and buried helix can be avoided. It results in no complications and gives good, reproducible aesthetic results. We recommend this technique as an alternative approach to managing the antihelix complex during otoplasty.

\section{REFERENCES}

[1] H. Tolleth, "Artistic Anatomy, Dimensions and Proportions of the External Ear," Clinics in Plastic Surgery, Vol. 5, No. 3, 1978, pp. 337-345.

[2] U. T. Hinderer, J. L. Del Rio and F. J. Fregenal, "Otoplasty for Prominent Ears,” Aesthetic Plastic Surgery, Vol. 11, No. 2, 1987, pp. 63-69.

[3] J. M. Converse, A. Nigro, F. A. Wilson, et al., "A Technique for Surgical Correction of Lop Ears,” Plastic and Reconstructive Surgery, Vol. 15, No. 5, 1955, pp. 411-418. doi:10.1097/00006534-195505000-00004

[4] A. J. McDowell, "Goals in Otoplasty for Protruding Ears," Plastic and Reconstructive Surgery, Vol. 41, No. 1, 1968, pp. 17-27. doi:10.1097/00006534-196801000-00004

[5] M. Spira, "Otoplasty What I Do Now-A 30-Year Perspective,” Plastic and Reconstructive Surgery, Vol. 104, No. 3, 1999, pp. 834-841.

[6] D. Furnas, "Correction of Prominent Ears with Multiple Sutures,” Clinics in Plastic Surgery, Vol. 5, No. 3, 1978, pp. 491-495.

[7] P. E. Johnson, "Otoplasty: Shaping the Antihelix," Aesthetic Plastic Surgery, Vol. 18, No. 1, 1994, pp. 71-74.

[8] D. W. Furnas, "Correction of Prominent Ears by Concha-Mastoid Sutures,” Plastic and Reconstructive Surgery, Vol. 42, No. 3, 1968, pp. 189-193. doi:10.1097/00006534-196809000-00001

[9] L. Caouette-Laberge, N. Guay, P. Bortoluzzi, et al., "Otoplasty: Anterior Scoring Techniques and Results in 500 Cases," Plastic and Reconstructive Surgery, Vol. 105, No.
2, 2000, pp. 504-515. doi:10.1097/00006534-196809000-00001

[10] A. Minderjahn, W. Huttl and H. Hildmann, "Mustarde’s otoplasty: Evaluation of Correlation between Clinical and Statistical Findings,” Journal of Maxillofacial Surgery, Vol. 8, No. 3, 1980, pp. 241-250. doi:10.1016/S0301-0503(80)80107-X

[11] A. H. Messner and W. S. Crysdale, "Otolplasty: Clinical Protocol and Long-Term Results," Archives of Otolaryngology_Head \& Neck Surgery, Vol. 122, No. 7, 1996, pp. 773-777. doi:10.1001/archotol.1996.01890190069016

[12] M. Spira, “Reduction Otoplasty,” In: R. M. Goldwyn, Ed., The Unfavorable Result in Plastic Surgery, Little Brown, Boston, 1984, pp. 307-323.

[13] O. J. Becker, "Surgical Correction of the Abnormally Protruding Ear," Archives of Otolaryngology-Head \& Neck Surgery, Vol. 50, No. 5, 1949, pp. 541-560. doi:10.1001/archotol.1949.00700010555002

[14] V. Chongchet, "A Method of Antihelix Reconstruction," British Journal of Plastic Surgery, Vol. 16, 1963, pp. 268272. doi:10.1016/S0007-1226(63)80120-4

[15] J. C. Mustarde, "The Correction of Prominent Ears Using Simple Mattress Sutures," British Journal of Plastic Surgery, Vol. 16, 1963, pp. 170-178. doi:10.1016/S0007-1226(63)80100-9

[16] P. Yugueros, J. A. Friedland and D. W. Furnas, “Otoplasty: The Experienceof 100 Consecutive Patients,” Plastic and Reconstructive Surgery, Vol. 108, No. 4, 2001, pp. 10451053. doi:10.1097/00006534-200109150-00038

[17] N. Horlock, A. Misra and D. Gault, “The Post Auricular Flap as an Adjunct to Mustarde and Furnas Type Otoplasty," Plastic and Reconstructive Surgery, Vol. 108, No. 6, 2001, pp. 1487-1491. doi:10.1097/00006534-200111000-00005

[18] S. Jeffery, "Complications Following Correction of Prominent Ears: An Audit Review of 122 Cases,” British Journal of Plastic Surgery, Vol. 52, No. 7, 1999, pp. 588-590. doi:10.1054/bjps.1999.3183

[19] J. C. Mustarde, "Correction of Prominent Ears Using Buried Mattress Sutures,” Clinics in Plastic Surgery, Vol. 5, No. 3, 1978, pp. 459-464.

[20] S. J. Stenstrom, "A Simple Operation for Prominent Ears," Acta Otolaryngologia, Vol. 224, 1966, p. 393.

[21] F. Peker and B. Celikoz, "Otoplasty: Anterior Scoring and Posterior Rolling Techniques in Adults," Aesthetic Plastic Surgery, Vol. 26, No. 4, 2002, pp. 267-273. doi:10.1007/s00266-002-2028-4

[22] S. J. Stenstrom, "A Natural Technique for Correction of Congenitally Prominent Ears," Plastic and Reconstructive Surgery, Vol. 32, 1963, pp. 509-518. doi:10.1097/00006534-196311000-00003

[23] P. A. Adamson, “Complications of Otoplasty,” Ear, Nose \& Throat Journal, Vol. 64, No. 12, 1985, pp. 668-574.

[24] B. W. Powell, "The Value of Head Dressings in the Postoperative Management of the Prominentear,” British Journal of Plastic Surgery, Vol. 42, No. 6, 1989, pp. 692-694. doi:10.1016/0007-1226(89)90083-0 